SCIENTIFIC LETTER

\title{
Amiodarone infusion in the treatment of acute atrial fibrillation or flutter: high versus low dose treatment
}

\author{
V Tuseth, H J Jaatun, K Dickstein
}

Heart 2005;91:964-965. doi: 10.1136/hrt.2004.049171

ntravenous amiodarone is a recognised treatment alternative for the conversion of atrial fibrillation to sinus rhythm. ${ }^{1-3}$ Consensus regarding dosage and duration of treatment has not been reached. ${ }^{45}$ Our coronary care unit routinely uses intravenous amiodarone for conversion of supraventricular arrhythmia in various clinical settings. Following a loading dose of $300 \mathrm{mg} / 30 \mathrm{mins}$, two alternative dosages of intravenous maintenance treatment $-50 \mathrm{mg} /$ hour and $100 \mathrm{mg} /$ hour-employed. We compared the efficacy and safety of these two regimens in an open randomised study.

\section{METHOD}

Patients with atrial fibrillation or flutter with duration of less than 48 hours were included. Patients with ECG evidence of transmural ischaemia, class III antiarrhythmic treatment within 14 days, cardiogenic shock, uncontrolled thyroid disease, previous symptomatic bradycardia without pacemaker, and tachycardia based on atrioventricular (AV) reentrant mechanisms were not included. Patients developing signs of haemodynamic decompensation were cardioverted.

The primary parameter was conversion to sinus rhythm. Secondary parameters were time to sinus rhythm and time to rate control. Heart rate, QT time, and PR interval at the time of conversion, were recorded continuously. Electrocardioversion before completion of the 24 hour period and interruption of study medication because of adverse experience were separate end points. Conduction abnormalities and any other adverse events during, and two hours following, the study were recorded. Eligible patients were included and allocated to high or low dose treatment in a single blinded manner according to a pre-specified allocation form. An intention to treat analysis was performed.

\section{RESULTS}

We acquired data from 50 patients in each group. The mean age was 68.5 years, 54 patients were male. Results for the primary end point significantly favoured the high dose group. A conversion rate of $80 \%$, compared to $60 \%$ in the low dose group, was achieved (fig 1).

From 10-24 hours of treatment a higher rate of conversion was recorded in the high dose group. Time to rate control did not differ significantly between the groups. A tendency towards lower heart rate, longer PR, and QT intervals was demonstrated in the high dose group. For two patients study treatment was stopped in the loading dose phase because of symptomatic hypotension and flushing shortly after initiation of treatment. In the low dose group electrocardioversion was performed before 24 hours in five patients. Reasons were: worsening heart failure, hypotension, and sustained tachycardia. In the high dose group, electrocardioversions were performed before study end because of persistent tachycardia in two patients. No other study patients required additional treatment for rate control. No patients developed symptomatic dysrhythmia requiring treatment during the trial. Peripheral lines were used for all patients and no

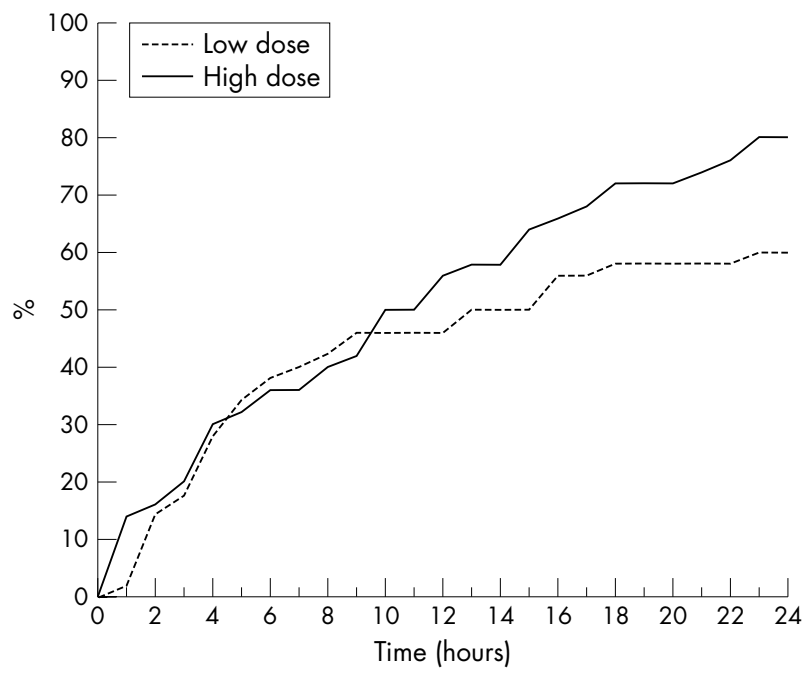

Figure 1 Kaplan-Meier diagram showing the rate of conversion during the study period $(n=100 ; p<0.05)$.

phlebitis was observed. The difference in response was most pronounced in the group with atrial flutter in that none of the six patients on low dose treatment converted, whereas all six patients in the high dose group converted.

\section{DISCUSSION}

Our results indicate superior efficacy with the higher dose of intravenous amiodarone for the routine treatment of acute atrial fibrillation or flutter in a coronary care unit setting. The divergence between the two groups after 10 hours suggests a sustained beneficial effect of prolonged high dose infusion as compared to low dose. The presence of severe co-morbidity in the majority of patients is considered pro-arrhythmic and would be expected to reduce the rate of conversion, and also increase the probability of recurrence of arrhythmia, as compared to that in more selected populations. Infections, heart failure, recent surgery, and myocardial ischaemia were the most common co-morbid conditions. Few patients needed cardioversion. No adverse event requiring specific treatment was seen. Both doses were effective for rate control. Only two patients received additional medication for rate control. As we wished to study the effect of amiodarone monotherapy to establish a simple routine treatment algorithm in a typical clinical setting, we did not include prespecified combined treatment strategies. An open label, single blind, alternate dose design was considered adequate to address our hypothesis, as the prescribed end points were objective. Selection bias was minimised as allocation was not known to the investigators before informed consent had been obtained. The equal distribution of flutter and fibrillation in the two groups indicates that preferential selection has not 
occurred. Having no placebo control group follows from our inclusion criteria in that rapid treatment was indicated on clinical grounds. In patients admitted to a coronary care unit, atrial fibrillation or flutter with tachycardia is a major cause of haemodynamic instability. Amiodarone has been established to have a rapid frequency modulating effect in addition to its efficacy with regard to conversion to sinus rhythm. It is most likely that the rate of conversion for both groups reflects an effect of the two treatment regimens evaluated. Our patients cannot be directly compared to populations in previous studies where patients with heart failure, recent myocardial infarction, pulmonary disease, and hypotension were generally not included. Such patients constituted a significant part of our material. A high spontaneous conversion rate has been reported in patients without such specified clinical predictors whereas conversion was notably diminished when these were present.

In our study, standard high dose intravenous amiodarone maintenance treatment following a loading dose of $300 \mathrm{mg} /$ 30 mins represents effective treatment for acute onset atrial fibrillation or flutter in the routine coronary care unit setting. The tendency towards increased late conversions in the high dose group suggests that amiodarone treatment should be continued for 24 hours before cardioversion in stabilised patients. A high dose, intravenous amiodarone regimen may represent an effective and safe strategy in the routine coronary care unit setting for treatment of atrial fibrillation or flutter.

The establishment of an effective routine treatment algorithm for the large and heterogeneous population seen in the coronary care unit would simplify clinical practice, however, further large scale studies are required to confirm our findings.

\section{Authors' affiliations}

V Tuseth, H J Jaatun, Cardiology Division, Stavanger University Hospital, Stavanger, Norway

K Dickstein, University of Bergen, Cardiology Division, Central Hospital of Rogaland

This study received financial support from the hospital research committee, and was approved by the regional ethics committee.

Correspondence to: Vegard Tuseth MD, Cardiology Division, Central Hospital of Rogaland, 4068 Stavanger, Norway; vtuseth@hotmail.com

Accepted 13 September 2004

\section{REFERENCES}

1 Hou ZY, Chang MS, Chen CY, et al. Acute treatment of recent-onset atrial fibrillation and flutter with a tailored dosing regimen of intravenous amiodarone. A randomised digoxin controlled study. Eur Heart J 1995; 16:521-8.

2 Clemo HF, Wood MA, Gilligan DM, et al. Intravenous amiodarone for acute heart rate control in the critically ill patient with atrial tachyarrhythmias. Am J Cardiol 1998; 11:594-8.

3 Cotter G, Blatt A, Kaluski E, et al. Conversion of recent onset paroxysmal atrial fibrillation to normal sinus rhythm: the effect of no treatment and high dose amiodarone. Eur Heart J 1999:20:1833-42.

4 Fuster V, Ryden LE, Asinger RW, et al. ACC/AHA/ESC guidelines for the management of patients with atrial fibrillation. Eur Heart $J$ 2001;22:1852-923.

5 Ikeda N, Nademanee K, Kannan R, et al. Electrophysiologic effects of amiodarone: experimental and clinical observation relative to serum and tissue drug concentrations. Am Heart J 1984;108:890-8.

\section{IMAGES IN CARDIOLOGY}

\section{Syncope in a 29 year old athlete: critical hint from the pulse watch}

A 9 year old man was admitted because of a single episode of syncope. He was previously healthy with no record of cardiovascular symptoms. He regularly engaged in different kinds of strenuous sporting activities including spinning. Spinning is a special setting of indoor cycling in a group, often led by an instructor. The music that accompanies the spinning is to push the cyclists to higher levels of performance. Usually people wear a heart rate monitor in form of a pulse wrist watch. After about 30 minutes of cycling the young man suddenly became unconscious. He regained consciousness after a minute and was brought to the emergency department of the nearest hospital. On admission the patient's physical status, ECG, and laboratory findings were unremarkable. However, the printout of the pulse watch (Polar 720) (panel A) showed not only the constant increase of the heart rate from around 90 beats/min to around 170 beats/min, but a sudden increase to around 225 beats/min when the patient became unconscious. This finding prompted admission of the patient to the hospital for further work up. An

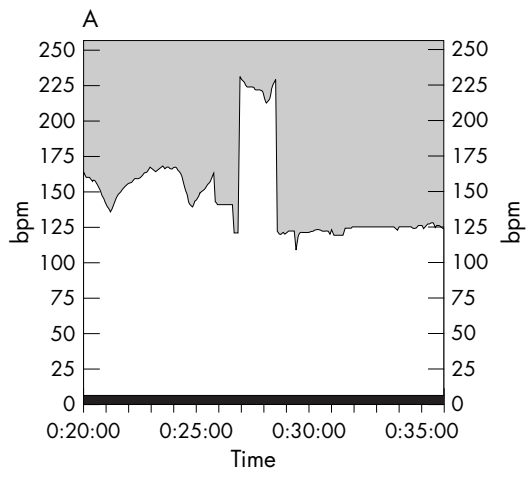

\begin{tabular}{|l|l|l|l|l|l|}
\hline Person & Name & & & Heart rate $\mathrm{mn}$ & $125 \mathrm{bpm}$ \\
\hline Date & 03.06 .2003 & Time & $17: 53: 03$ & Heart rate $\mathrm{mx}$ & $231 \mathrm{bpm}$ \\
\hline Sports & Spinning & Duration & $2: 14: 31$ & & \\
\hline Event & Syncope & Selection & $0: 36: 00$ & & \\
\hline
\end{tabular}

electrophysiologic study revealed the easy and reproducible induction of monomorphic ventricular tachycardia (VT). Coronary angiography revealed unexpected severe three vessel coronary artery disease (panel B). The patient underwent coronary bypass graft surgery. Subsequent electrophysiologic study revealed the same VT induction,

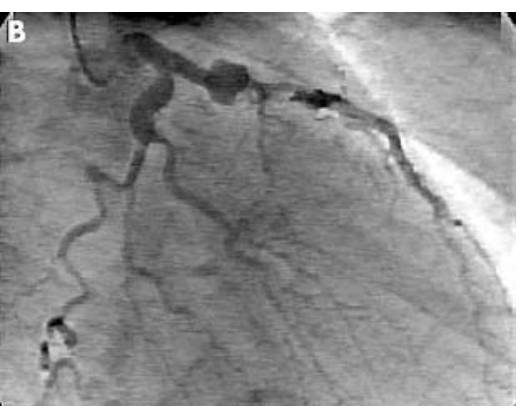

prompting the implantation of an implantable cardioverter-defibrillator.

It is very possible that without the findings from the pulse watch, the work up of this young man's syncope would have been less straightforward, and would not have uncovered the mechanism of the syncope-VT-or the underlying coronary artery disease. The critical information provided by the pulse watch may well have saved the patient's life.

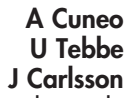
alessandro.cuneo@klinikum-lippe.de 